\title{
Microcantilever based disposable viscosity sensor for serum and blood plasma measurements
}

\author{
Onur Cakmak ${ }^{\mathrm{a}}$, Caglar Elbuken ${ }^{\mathrm{b}, 1}$, Erhan Ermek ${ }^{\mathrm{b}}$, Aref Mostafazadeh ${ }^{\mathrm{b}}$, Ibrahim Baris ${ }^{\mathrm{c}}, \mathrm{B}$. Erdem Alaca ${ }^{\mathrm{a}}$, \\ Ibrahim Halil Kavakli c,d, Hakan Urey ${ }^{\mathrm{b}, *}$ \\ ${ }^{a}$ Koc University, Mechanical Engineering, Rumeli Feneri Yolu, 34450 Sariyer, Istanbul, Turkey \\ ${ }^{\mathrm{b}}$ Koc University, Electrical and Electronic Engineering, Rumeli Feneri Yolu, 34450 Sariyer, Istanbul, Turkey \\ ' Koc University, Department of Molecular Biology and Genetics, Rumeli Feneri Yolu, 34450 Sariyer, Istanbul, Turkey \\ ${ }^{\mathrm{d}}$ Koc University, Chemical and Biological Engineering, Rumeli Feneri Yolu, 34450 Sariyer, Istanbul, Turkey
}

\section{A R T I C L E I N F O}

Article history:

Available online 20 July 2013

\section{Keywords:}

Viscosity

Cantilever

Microcantilever

MEMS

Blood

Blood Plasma

Serum

Magnetic actuation

\begin{abstract}
A B S T R A C T
This paper proposes a novel method for measuring blood plasma and serum viscosity with a microcantilever-based MEMS sensor. MEMS cantilevers are made of electroplated nickel and actuated remotely with magnetic field using an electro-coil. Real-time monitoring of cantilever resonant frequency is performed remotely using diffraction gratings fabricated at the tip of the dynamic cantilevers. Only few nanometer cantilever deflection is sufficient due to interferometric sensitivity of the readout. The resonant frequency of the cantilever is tracked with a phase lock loop (PLL) control circuit. The viscosities of liquid samples are obtained through the measurement of the cantilever's frequency change with respect to a reference measurement taken within a liquid of known viscosity. We performed measurements with glycerol solutions at different temperatures and validated the repeatability of the system by comparing with a reference commercial viscometer. Experimental results are compared with the theoretical predictions based on Sader's theory and agreed reasonably well. Afterwards viscosities of different Fetal Bovine Serum and Bovine Serum Albumin mixtures are measured both at $23^{\circ} \mathrm{C}$ and $37^{\circ} \mathrm{C}$, body temperature. Finally the viscosities of human blood plasma samples taken from healthy donors are measured. The proposed method is capable of measuring viscosities from $0.86 \mathrm{cP}$ to $3.02 \mathrm{cP}$, which covers human blood plasma viscosity range, with a resolution better than $0.04 \mathrm{cP}$. The sample volume requirement is less than $150 \mu \mathrm{l}$ and can be reduced significantly with optimized cartridge design. Both the actuation and sensing are carried out remotely, which allows for disposable sensor cartridges.
\end{abstract}

(c) 2013 Published by Elsevier Inc.

\section{Introduction}

Blood plasma viscosity can be used for the diagnosis of several diseases such as cardiovascular disorders, rheumatoid arthritis, certain autoimmune diseases [1]. Plasma viscosity increases in parallel with erythrocyte sedimentation rate (ESR), however, it is not influenced by hematocrit (anemia, polycytemia). Therefore, for hyperviscosity syndromes plasma viscosity can be more useful than ESR, which is widely used for diagnostics. The blood plasma viscosity range for healthy individuals is between $1.1 \mathrm{cP}$ and $1.3 \mathrm{cP}$. It can be elevated up to $3 \mathrm{cP}$ in symptoms of hyperviscosity [2]. Current devices for blood plasma viscosity measurement have limitations as reviewed below. This paper reports a fast and conve-

\footnotetext{
* Corresponding author. Tel: +90212334 1772.

E-mail addresses: ocakmak@ku.edu.tr (O. Cakmak), hurey@ku.edu.tr (H. Urey).

${ }^{1}$ Present address: Bilkent University, UNAM, National Nanotechnology Research Center, Bilkent, 06800 Ankara, Turkey.
}

nient novel measurement method for measuring blood plasma and serum viscosity from low sample volumes with a high sensitivity.

There are several approaches for viscosity measurements. Viscometers based on the rolling ball (or falling sphere) principle have a wide usage for various liquids. However, these instruments require custom-designed, precision-manufactured, long capillary tubes. These capillary tubes are costly and require cleaning after each use, thus not very suitable for medical use. Another common viscometer is based on rotating tube principle [3] which is generally used as a bench top tool requiring high sample volumes. Capillary-tube viscometers are being used to measure the plasma or serum viscosity $[4,5]$ but suffer from cleaning requirement after each use which can be challenging due to the capillary geometry. Hence, there is a need for a viscometer which has disposable cartridges requiring low sample volume in order to facilitate blood plasma viscosity measurements in Point of Care (POC) tests.

The dynamic behavior of resonant microscale structures strongly depend on the medium in which they are operating [6]. Hence, this permits the measurement of the rheological properties 
of that medium by tracking the changes in resonant frequency and quality factors (Q). Different types of micro-mechanical viscosity sensors based on various techniques have been reported. These include, measurement of the torsional vibration of an oscillating tube, thickness shear mode resonators, acoustic wave sensors and oscillating plate/membrane structures [7-12]. Cantileverbased viscosity sensors, as discussed in this paper, also have a wide usage. Viscosity measurements of engine oils, silicone oils and glycerol solutions with the use of millimeter scale cantilevers with large sample volumes (around $3 \mathrm{ml}$ ) have been demonstrated [1315]. Although there are other studies introducing measurements with microcantilevers [16-22], the working range or sensitivity is not suitable for the blood plasma viscosity measurement due to various limitations imposed by operating cantilevers in liquids.

MEMS cantilever based viscosity sensors have been previously designed specifically for glucose concentration detection [23-26]. Glucose levels are detected based on affinity binding of glucose sensitive polymeric matrix encapsulated over the sensor. Although these studies provide performance metrics of the sensor for various glucose solutions, the complete analysis of the sensor as a viscometer is not reported.

There are also commercial viscometers (Rheosense $\mu$ Visc [27] and Gluconline [28]) which utilize single-use cartridges and eliminate any requirements of cleaning. The sample is pumped inside a channel and the pressure difference along the channel is measured at two different locations by pressure sensors. The pressure drop between these two locations is used to calculate the viscosity of the sample. The primary downside of these systems is that they require bubble-free sampling or bubble trappers since bubbles cause additional pressure drop.

The microcantilevers and the sensing method developed in this paper offer a novel method for fast and convenient blood plasma viscosity measurement with high resolution in a cartridge suitable for single use. In the first part, MEMS fabrication and principle of the operation are presented. Afterwards the analytical approximations used during the assessment of the experimental results are shown. In the results and discussion section, repeated measurement results with different glycerol solutions at different temperatures are shown in addition to the measurements with Fetal Bovine Serum (FBS) and blood plasma samples. The comparison of these results with commercial viscometers is also presented.

\section{Materials and methods}

\subsection{Microfabrication}

The viscometer device utilizes a microfabricated chip containing electroplated nickel microcantilevers. These Ni cantilevers are fabricated with a single mask process [29,30]. A 20/100 nm Cr/Au layer is deposited on a $4^{\prime \prime}$ diameter, $<100>$ single crystalline silicon prime wafer (with thickness and resistivity values of $400 \pm 25 \mu \mathrm{m}$ and $0.1-0.5 \Omega \mathrm{cm}$, respectively) using RF sputtering with Vaksis PVD Handy sputter tool. The wafers were provided from Silicon Quest Inc. (LOT No: p0809696). Sputtering is conducted inside Ar plasma at $1.5 \mathrm{e}-5$ Torr vacuum. The $\mathrm{Cr}$ target (K.J. Lesker Inc. part no: EJTCRXX353A2) used during sputter has a $3^{\prime \prime}$ diameter, $0.125^{\prime \prime}$ thickness and $99.95 \%$ purity whereas the Au target (K.J. Lesker Inc. part no: EJTAUXX403A2) has same dimensions and $99.99 \%$ purity. Here $\mathrm{Cr}$ layer is used to provide adhesion of gold layer to $\mathrm{Si}$, whereas Au layer serves as the seed layer for subsequent Ni electroplating (Fig. 1b). A positive photoresist (PR), AZ1514H ${ }^{\circledR}$ (Microchemicals GmbH. Batch: DEAZ062154-A7) with a thickness of $1500 \mathrm{~nm}$, layer is coated on the top of Au surface with SPS Spin150 manual spin-coater and patterned with UV lithography using KarlSüss MA45 mask aligner. Inside the mask aligner, the wafer is ex- posed to UV light whose intensity is $22 \mathrm{~mW} / \mathrm{cm}^{2}$. The exposure time is determined as $17 \mathrm{~s}$ with contact printing option. After the development with AZ351B Developer (Microchem. batch: DEAZ056342) in $1 \mathrm{~min}$, the electroplating of nickel is conducted with a custom made setup using a nickel sulphamate bath at $45^{\circ} \mathrm{C}$ with $5 \mathrm{~mA} / \mathrm{cm}^{2}$ current density. The bath is composed of $600 \mathrm{~g} / \mathrm{L}$ nickel sulphamate $\mathrm{Ni}\left(\mathrm{SO}_{3} \mathrm{NH}_{2}\right)_{2} \cdot 4 \mathrm{H}_{2} \mathrm{O}$ ) (Sigma Aldrich 262277 Lot No:57664842), $10 \mathrm{~g} / \mathrm{L}$ nickel chloride $\left(\mathrm{NiCl}_{2} \cdot 6 \mathrm{H}_{2} \mathrm{O}\right)$ (Alfa Aesar A14366 No: 61100188) and $40 \mathrm{~g} / \mathrm{L}$ boric acid $\left(\mathrm{H}_{3} \mathrm{BO}_{3}\right)$ (Alfa Aesar A16624 No: 07IO60004). An Inco $4 \times 4^{\prime \prime}$ type Nickel anode with purity larger than $99.9 \%$ is used during electroplating.

After the electrodeposition of nickel layer, the remaining PR is stripped via AZ100 Remover (Microchem. batch: DEAZ055450). Wet etching of $\mathrm{Au}$ and $\mathrm{Cr}$ are conducted with 1:3 dilution of Transene Inc. GE- 8148 (LOT No: 081103) type gold etchant and 1:3 dilution of Microchrome Inc. CEP-200 chromium etchant (LOT No: 163021) at room temperature in $90 \mathrm{~s}$ and $75 \mathrm{~s}$, respectively. Finally, cantilevers are released through wet etching of Si in $35 \% \mathrm{KOH}$ solution (Sigma Aldrich No: 435125 ) at $60^{\circ} \mathrm{C}$ in $45 \mathrm{~min}$. Anisotropic wet etching of silicon is carried out to a depth of $15 \mu \mathrm{m}$ to avoid squeeze film damping effect. The finished cantilevers have a length of $60 \mu \mathrm{m}$, excluding the head structure consisting of gratings, a width of $5 \mu \mathrm{m}$ and a thickness of $1 \mu \mathrm{m}$. The diffraction gratings at the tip have $3 \mu \mathrm{m}$ width, $20 \mu \mathrm{m}$ length and the grating period is $6 \mu \mathrm{m}$. The close-up micrograph and the 3D drawing of the cantilevers can be seen in Fig. 1a.

After microfabrication, the chip containing microcantilevers is attached inside a microfluidic chamber by epoxy. The chamber is formed by machining $2 \mathrm{~mm}$ thick Poly Methyl Methacrylate (PMMA) plaque which is provided from local manufacturer. The channel depth is $500 \mu \mathrm{m}$ and the total volume of the microchamber is $150 \mu$ l. The chamber is machined in water drop geometry (Fig. 2) in order to prevent sharp corners which cause bubbling during liquid exchange. It should be noted that the sample volume requirement can be further reduced for a specific application. The chips are designed for research use and pilot produced. Hence, they have a large real estate $(10 \times 10 \mathrm{~mm})$ for ease of handling. The size of the chip can be reduced down to $2 \times 2 \mathrm{~mm}$ and the required sample volume can be $10 \mu \mathrm{l}$ for that kind of a design. The chamber has a single inlet and outlet with drilled holes for fluidic access. PMMA base is sealed by a standard glass slide using epoxy bonding (Fig. 2).
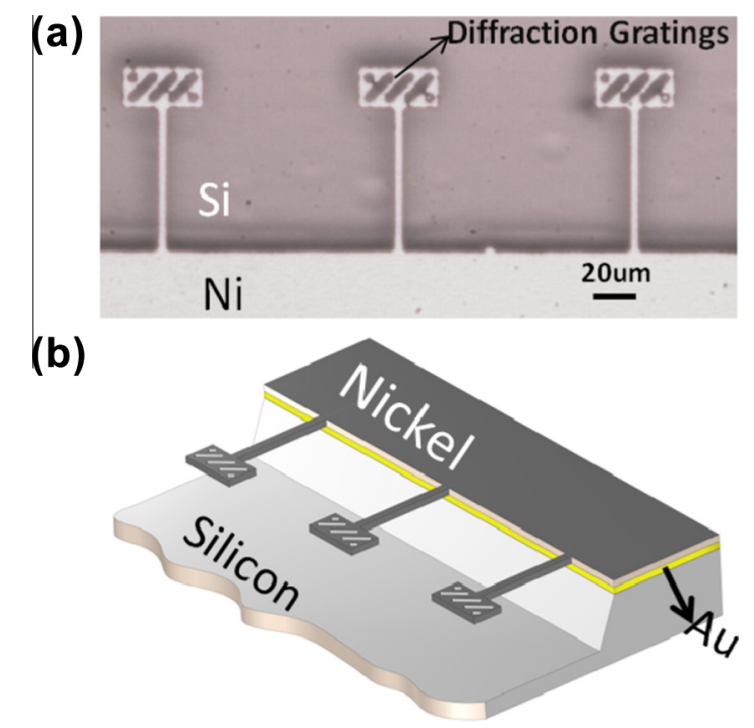

Fig. 1. (a) Micrograph of microfabricated cantilevers with diffraction gratings. (b) 3D illustration of the cantilevers, which are $15 \mu \mathrm{m}$ above the Si surface. 


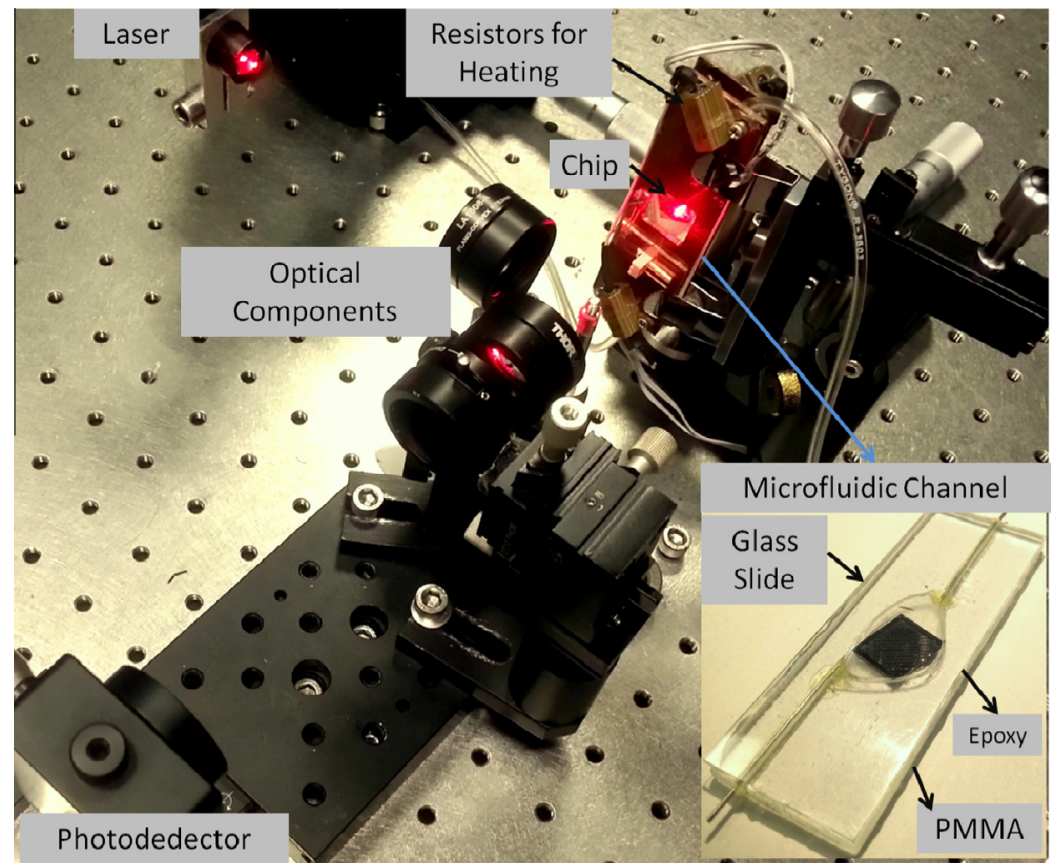

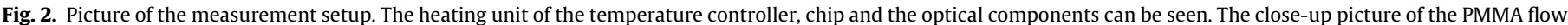
cell is at the bottom right. The glass slide is glued on top of the PMMA flow cell with epoxy to provide sealing.

\subsection{Measurement principle and setup}

\subsubsection{Magnetic actuation}

The nickel cantilevers are actuated magnetically with a coil attached to the backside of the PMMA flow cell as shown in Fig. 2. A pair of bar $(3 \times 4 \times 9 \mathrm{~mm})$ neodymium permanent magnet is placed parallel to the cantilever in order generate DC magnetic field $(0.5 \mathrm{mT})$ to saturate magnetization of the Ni cantilevers. For minimizing parasitic capacitive effects and electric resonances in the coil itself, a low turn coil $(N=80)$ is used. The total diameter and height of this custom made coil is $14 \mathrm{~mm}$ and $12 \mathrm{~mm}$ respectively, whereas the wire diameter is $0.3 \mathrm{~mm}$. The coil is driven with a high power broadband current amplifier with a fixed current of $1 A_{p-p}$ over the whole frequency range. Since no direct electrical contacts are required on the chip, magnetic actuation with an external coil has a significant advantage for in-liquid operation.

\subsubsection{Optical read-out}

Sensing is also carried out remotely by optical interferometric readout system (Fig. 3). An interference pattern is formed by laser beams reflecting from top Ni surface (movable cantilever) and bottom Si surface (fixed reference) (Fig. 1b). This leads to an intensity modulation on the photodiode in response to the deflection of $\mathrm{Ni}$ cantilever with respect to the Si reference surface. Using the intensity modulation of the first diffraction order, the phase and frequency of the cantilever can be monitored [29,30]. Optical detection is carried out using a HITACHI $15 \mathrm{~mW}, 635 \mathrm{~nm}$ type laser diode, diffraction gratings embedded at the tip of each cantilever, a THORLABS PDA36A-EC type photodetector, and lenses. The entire readout electronics can be miniaturized and made portable.

\subsubsection{Resonance frequency tracking}

In order to observe the frequency changes due to viscosity, a custom-made PLL-based closed-loop system is used. The system consists of a broadband mixed signal PLL circuit which locks the system to any desired phase difference between the actuation and the readout signal. The resonance frequency tracking system is specially designed to operate with low $Q$ systems and high back- ground noise levels. Using the phase for resonant tracking is preferred as amplitude based tracking proved to be noisier and affected by other parameters such as laser amplitude noise during the measurements As the quality factors of the cantilevers are very low (around 1.5), the resonance occurs around $60^{\circ}$ phase difference as shown in Fig. 4. Thus the PLL system is designed to track the resonance frequency at $60^{\circ}$ phase difference for achieving maximum readout amplitude and signal to noise ratio (SNR). The frequency of the system is monitored with Tektronix FCA 3100 Frequency Counter to average several million cycles within a few seconds and allows sub-Hz frequency sensitivity even in liquids. The equipment is capable of measuring frequency with 12 digit resolution up to $300 \mathrm{MHz}$, and the time resolution is $50 \mathrm{ps}$. The device is connected to LabVIEW (NI, 2011) interface via USB port in order to record real-time data. A custom Labview interface is developed which records the real-time frequency data in a common data format. In Fig. 5 each data point shows the average of the values acquired during $1 \mathrm{~s}$ time interval. In order to achieve higher SNR and avoid transient condition changes the average of 200 data points is used. The standard deviation of these 200 data points is in the order of $0.01 \%$.

\subsubsection{Temperature control}

Temperature is a critical parameter when viscosity measurements are considered. The temperature change has an effect on viscosity and density of the fluid and elastic properties of the cantilever structure. The variation of these parameters results in a change of the resonant frequency. The change in viscosity of the fluid is the dominant parameter causing this frequency shift [31]. In order to limit temperature variations of the system an analog temperature controller system is added to the system. It consists of a temperature sensor, AD590, a controller circuit and four resistors for heating positioned symmetrically outside the flow cell (Fig. 2). Two copper layers are attached on front and back walls of the flow cell to conduct heat to the system in a uniform manner. AD590 provides a linear temperature reading over its full range. Using this controller system the temperature of the fluid can be set to a precision of $0.1^{\circ} \mathrm{C}$. 


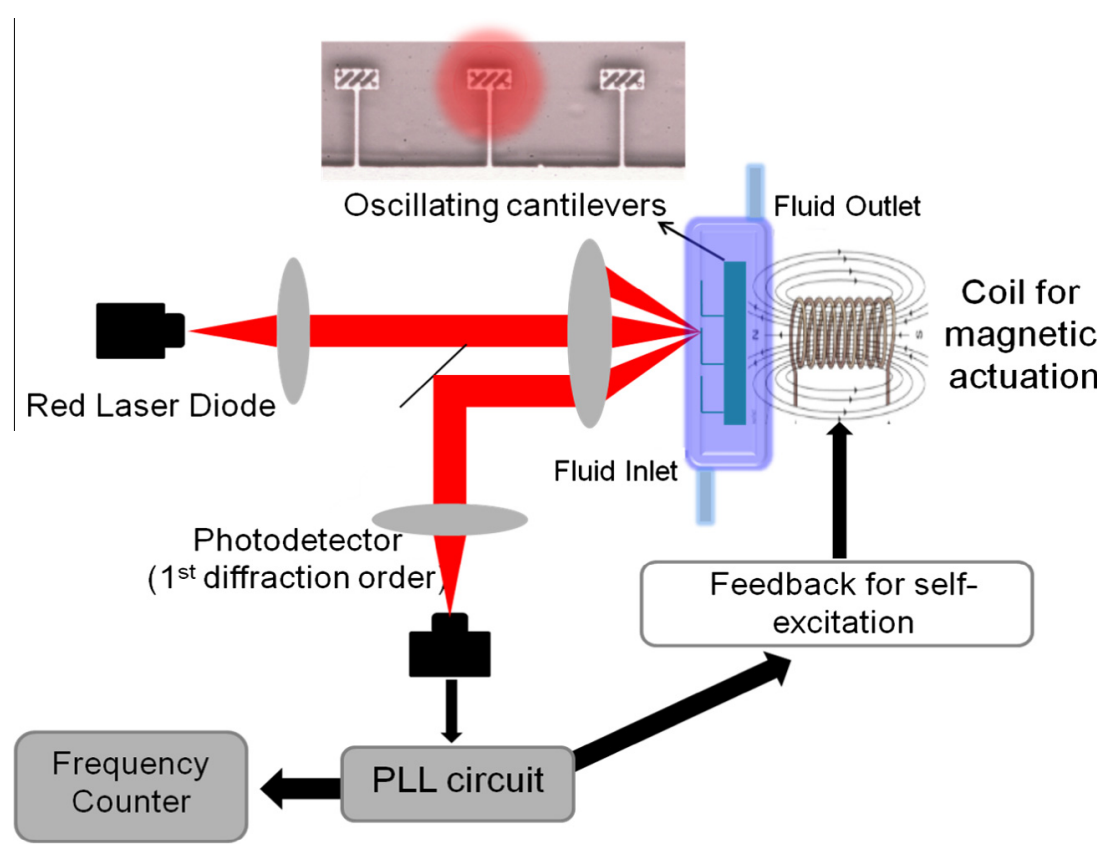

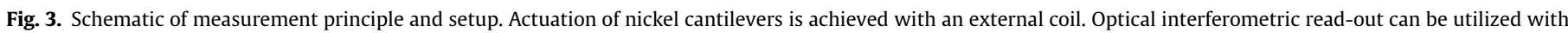
diffraction gratings at the tip of each cantilever. The closed-loop system with phase-lock loop (PLL) circuit enables resonance frequency tracking.

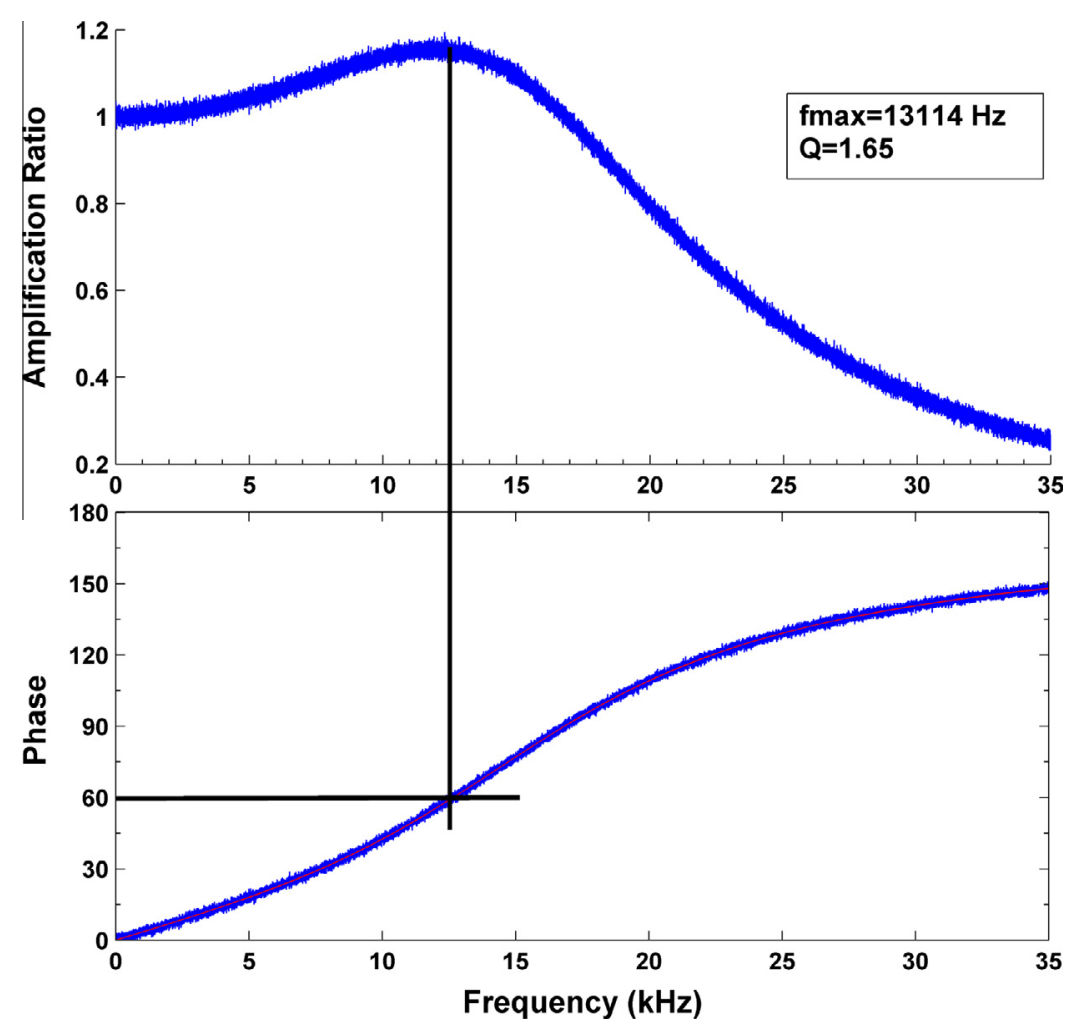

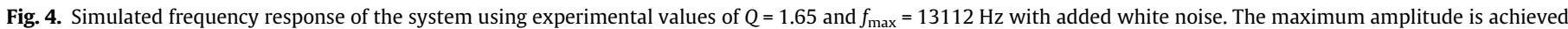
at $60^{\circ}$ phase difference which is the value that the system is locked by PLL.

\section{Theoretical background}

The cantilever beam structure is considered as Euler-Bernoulli beam. When the cantilever is operating in viscous media, a hydrodynamic force per unit length, $F_{\text {fluid, }}$ is applied to the structure [6]. This force includes two different terms [17]:

$$
F_{\text {fluid }}=-g_{1} \dot{\omega}-g_{2} \ddot{\omega}
$$

$\omega=\omega(x, t)$ is the deflection function of the beam axis. The first term is the viscous drag force and second term is the inertial force related to the liquid mass accelerated by the cantilever as shown in Fig. 6a. According to Sader's theory [6] the liquid vibrating with the canti- 


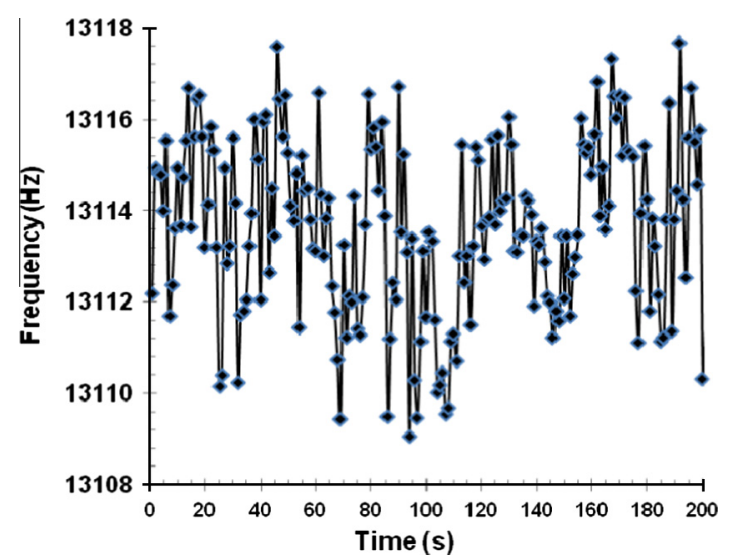

Fig. 5. The frequency data obtained with PLL based closed-loop system. The average of 200 data points (200 s.) is $13113.67 \mathrm{~Hz}$ and standard deviation is $1.89 \mathrm{~Hz}$.
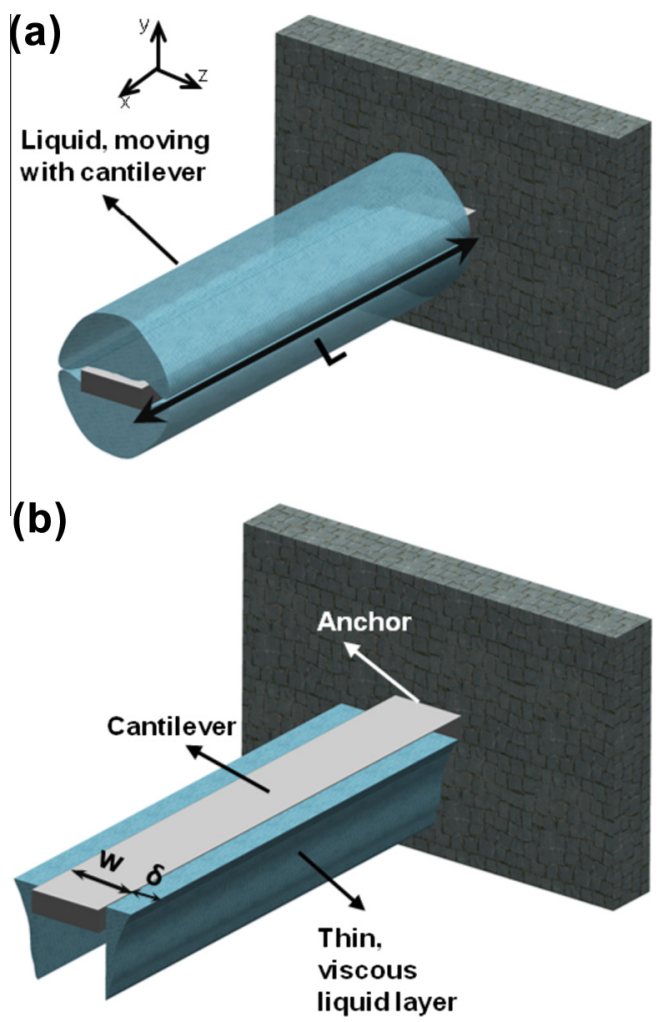

Fig. 6. (a) The illustration of the liquid mass, $\mathrm{Lg}_{2}$, accelerated by the vibrating cantilever. (b) Thin viscous layer, $\delta$, where fluid velocity dropped by a factor of $1 / e$ during cantilever motion.

lever can be assumed as a cylinder with mass per length as given by g2. g1 and g2 can be expressed as [19]:

$g_{1}=M_{L}\left(2 \pi f_{L}\right)\left(b_{1} \frac{\delta}{w}+b_{2}\left(\frac{\delta}{2}\right)^{2}\right)$

$g_{2}=M_{L}\left(a_{1}+a_{2} \frac{\delta}{w}\right)$

where $w$ is the width of the cantilever, $f_{L}$ is the damped resonant frequency of the cantilever in liquid. $a_{1}=1.0553, a_{2}=3.7997$, $b_{1}=3.8018$, and $b_{2}=2.7364$ are dimensionless "Maali's parameters" for an infinitely thin beam [32]. $M_{L}=\pi \rho_{f} w^{2} / 4$, where $\rho_{f}$ is the fluid density. The illustration in Fig. 6b shows the $\delta$ parameter, which is the fluid layer thickness where the fluid velocity drops by a factor of $1 / e . \delta$ can be expressed using the viscosity of the fluid $(\mu)$, as:

$\delta=\sqrt{\frac{2 \mu}{\rho_{f}\left(2 \pi f_{L}\right)}}$

As an example, using the viscosity and density of deioinized (DI) water, $\delta / w$ ratio becomes 0.213 . This value is calculated using an effective width for our cantilever design with varying width. The resonant frequency and $Q$ including the hydrodynamic force effects in liquid can be expressed as:

$f_{L}=f_{0} \frac{1}{\sqrt{1+L g_{2} / m}} \sqrt{1-\frac{1}{2 Q^{2}}}$

$Q=2 \pi f_{0} \frac{\sqrt{1+L g_{2} / m}}{L g_{1} / m}$

$L$ and $m$ are the length and mass of the cantilever respectively, and $f_{0}$ is the undamped resonant frequency in vacuum, which is very close to that of air. The mass of the cantilever is calculated as $8.8 \mathrm{ng}$ whereas the added mass, $\mathrm{Lg}_{2}$ is $32.5 \mathrm{ng}$ in DI water.

Frequency shift due to viscosity and density changes can be computed iteratively using the above equations. The experimental values for fluid density and viscosity, and resonant frequency in air, $f_{0}$, are substituted in Eqs. $(2,3,4,5)$ to calculate $\delta, g_{1}$ and $g_{2}$, and $f_{L}$. As can be seen from Eq. 4 and 5 ; the $\delta$ the $f_{L}$ are dependent on each other. Hence an iterative solution is required to obtain these values. The resonant frequency in air, $f_{0}$, is chosen as the initial value of $f_{\mathrm{L}}$. The iteration continues until the difference is less than $1 \mathrm{e}-$ $4 \mathrm{~Hz}$ between two successive values of $f_{\mathrm{L}}$. The number of iterations required to converge is about 9 .

\section{Results and discussion}

\subsection{Measurements with glycerol solutions}

During the experiments deionized (DI) water at room temperature $\left(23^{\circ} \mathrm{C}\right)$ is used as the reference liquid. Glycerol/DI solutions with varying glycerol concentrations have been used in order to calibrate the system and obtain its response to viscosity changes. The viscosities and densities of glycerol solutions used in the experiments can be seen in Table 1 . In order to cover the viscosity range of blood plasma, glycerol solutions between $5 \%$ and $40 \%$ are used which correspond to viscosity range of 1.06-3.02 cP. Viscosity values presented in Table 1 are obtained by the measurements with a reference commercial viscometer, Anton Paar AMVn. This device works based on rolling ball principle: a gold-coated steel ball is introduced inside a glass capillary filled with sample. The time taken for the ball to roll a fixed distance between two magnetic sensors is converted into viscosity measurements. A temper-

Table 1

Viscosities and densities of each glycerol solution used in experiments. The values are obtained with reference commercial viscometer, Anton Paar AMVn.

\begin{tabular}{lll}
\hline Glycerol Percentage (\%) & Viscosity $(\mathrm{cP})$ & Density $\left(\mathrm{kg} / \mathrm{m}^{3}\right)$ \\
\hline 0 & 0.9532 & 0.9977 \\
5 & 1.0642 & 1.0095 \\
10 & 1.1698 & 1.0214 \\
12 & 1.2323 & 1.0266 \\
14 & 1.2685 & 1.0312 \\
15 & 1.3040 & 1.0337 \\
20 & 1.4838 & 1.0462 \\
30 & 2.2843 & 1.0717 \\
40 & 3.0221 & 1.0983 \\
\hline
\end{tabular}




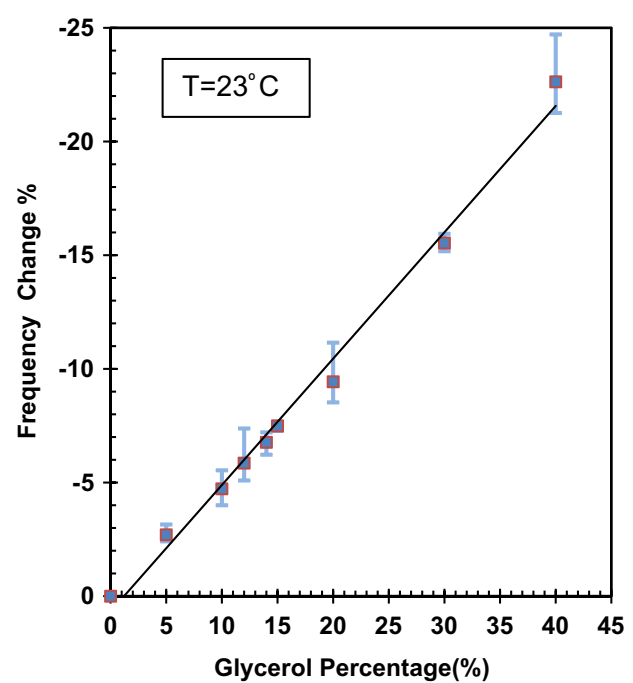

Fig. 7. Percent frequency change obtained from different glycerol solutions at $23^{\circ} \mathrm{C}$, room temperature. The data points show the averages of four different measurements for each glycerol solution $(5 \%, 10 \%, 12 \%, 14 \%, 15 \%, 20 \%, 30 \%$, and $40 \%)$. The error bars show the minimum and maximum values among these measurements. The line is the linear trendline fit to this data.

ature sensor close to the capillary's surface measures the temperature within the capillary block.

Measurement process with microcantilevers is as follows: First, the microchannel is filled with DI water and resonance frequency is recorded, then DI water is drained and the sample liquid is pumped into the channel and the frequency value is recorded again [33]. The first frequency is used as reference and the percent frequency change is calculated with respect to that reference. Same procedure is repeated for the next solution. The process is repeated at least four times for each solution. The frequency change with respect to DI water for each glycerol solution is shown in Fig. 7.

Viscosity values for each glycerol solution are obtained using Table 1 and the experimental frequency shift measurements are plotted in Fig. 8. A 2nd order polynomial is used for curve-fitting the experimental data with good accuracy. The fit function is used as a calibration curve for the subsequent measurements. For a given solution, percentage frequency change is measured and the corresponding viscosity value is calculated using the fit function.
All of the MEMS viscosity measurement results in this paper are based on the experimental data and the fit function. In order to check the validity of the analytical formulas, theoretical predictions are also shown in Fig. 8. The slight discrepancy can be associated with the assumptions used during the analytical approach [6] and experimental errors.

The variation between repetitive measurements of the same sample is also shown with the error bars. Although they are in an acceptable region, it can be asserted that experimental error during the liquid exchange and sample preparation and local heating of the sample with focused laser spot give rise to this variation.

The viscosities of the glycerol solutions with $10 \%, 12 \% 14 \%$ and $15 \%$ is prepared in order to observe the sensitivity of the system. The system is capable of resolving $0.04 \mathrm{cP}$ viscosity difference which is the difference between $14 \%$ and $15 \%$ glycerol solutions. To the best of our knowledge this is the best sensitivity value reported with a MEMS viscometer working in this low viscosity range.

\subsection{Measurements at different temperatures}

Increase in temperature results in a decrease in viscosity leading to an increase in the resonant frequency of the system. Also it should be noted that blood plasma and serum measurements should be conducted at $37{ }^{\circ} \mathrm{C}$ body temperature, hence, reliability of the temperature control must also be confirmed. For this purpose viscosity of the $15 \%$ glycerol solution is measured at 23,25 , 30,35 and $40^{\circ} \mathrm{C}$. The viscosity values obtained with MEMS viscometer and reference device are presented in Fig. 9. The values taken with the MEMS viscometer is slightly smaller than the reference measurements (Table 2). This can be attributed to the influence of the local laser heating. The small volume around the cantilever can have slightly higher temperature than other portions due to laser heating. It is extremely challenging to measure local temperature variation in a given liquid at micro scale, therefore this hypothesis is not backed up with experimental data.

\subsection{Measurements with FBS and BSA solutions}

Before measuring human plasma viscosity, viscosities of Fetal Bovine Serum, FBS (Sigma-Aldrich F2442, and Bovine Serum Albumin, BSA, (Sigma-Aldrich A3675) solutions were measured. By

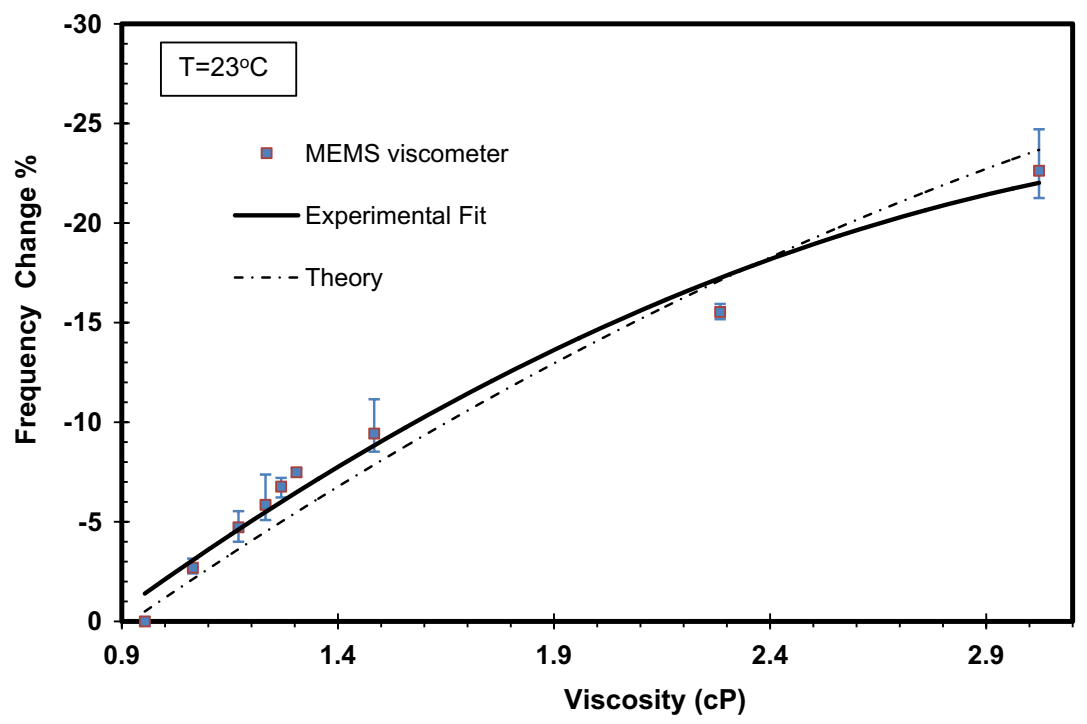

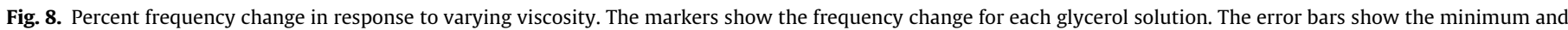

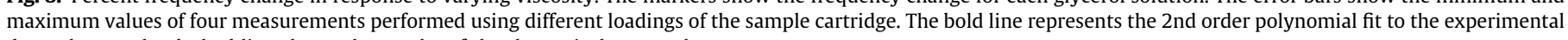
data whereas the dashed line shows the results of the theoretical approach. 


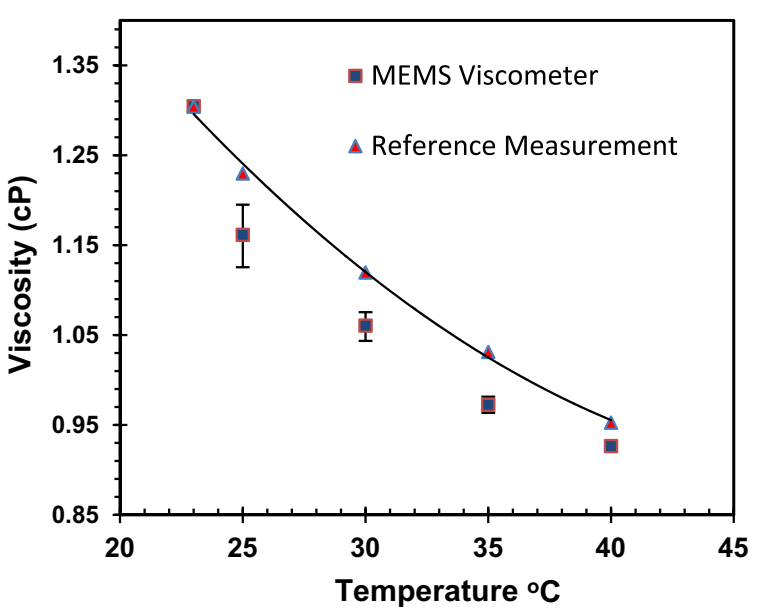

Fig. 9. Frequency change due to temperature increase. Viscosities of $15 \%$ glycerol solutions at $23^{\circ} \mathrm{C}, 25^{\circ} \mathrm{C}, 30^{\circ} \mathrm{C}, 35^{\circ} \mathrm{C}$ and $40^{\circ} \mathrm{C}$ are measured with both reference commercial viscometer and proposed MEMS viscometer. Error bars represent standard deviation of 200 data points during one experiment.

Table 2

Viscosities of $15 \%$ glycerol solution at different temperatures measured with both reference commercial viscometer and MEMS viscometer.

\begin{tabular}{llllll}
\hline Temperature $\left({ }^{\circ} \mathrm{C}\right)$ & $\begin{array}{l}\text { Viscosity }(\mathrm{cP}) \\
\text { Reference } \\
\text { Measurement }\end{array}$ & $\sigma(\mathrm{cP})$ & $\begin{array}{l}\text { Viscosity } \\
(\mathrm{cP}) \text { MEMS } \\
\text { viscometer }\end{array}$ & $\begin{array}{l}\sigma \\
(\mathrm{cP})\end{array}$ & $\begin{array}{l}\% \\
\text { difference }\end{array}$ \\
\hline 23 & 1.304 & 0.0008 & 1.303 & 0.006 & -0.07 \\
25 & 1.219 & 0.0010 & 1.161 & 0.033 & 4.75 \\
30 & 1.119 & 0.0008 & 1.060 & 0.017 & 5.27 \\
35 & 1.031 & 0.0011 & 0.972 & 0.009 & 5.72 \\
40 & 0.952 & 0.0008 & 0.926 & 0.006 & 2.73 \\
\hline
\end{tabular}

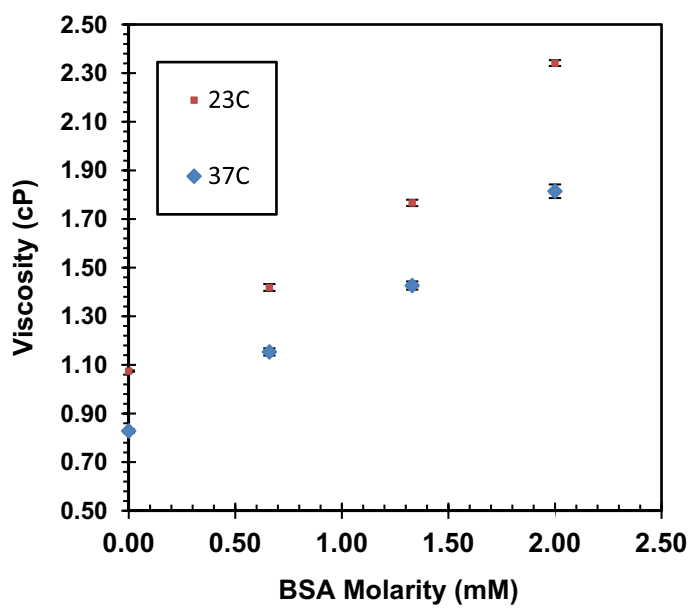

Fig. 10. Viscosities of Bovine Serum Albumin (BSA) solutions ( $0 \mathrm{M}$ (only FBS) $0.66 \mathrm{mM} 1.33 \mathrm{mM}, 2 \mathrm{mM}$ ) measured with MEMS viscometer. Measurements at room temperature and body temperature are shown. The error bars show the standard deviation of 200 data points during one experiment which can be named as the "noise floor" of the system.

adding BSA into FBS the viscosity can be increased significantly whereas the density is not affected noticeably. This makes BSAFBS solutions a good and easily accessible laboratory model of healthy/unhealthy human plasma. The hyperviscosity syndrome shows up around $1.8 \mathrm{cP}[2]$. In order to cover that range, BSA-FBS solutions with different molarities are prepared. The molecular weight of BSA is $66.5 \mathrm{kDa}$. The viscosities of different solutions
Table 3

Viscosities of Serum and BSA solutions measured with both reference commercial viscometer and MEMS viscometer at room temperature and body temperature.

\begin{tabular}{llllll}
\hline $\begin{array}{l}\text { Solution } \\
\text { Type }\end{array}$ & $\begin{array}{l}\text { Temperature } \\
\left({ }^{\circ} \mathrm{C}\right)\end{array}$ & $\begin{array}{l}\text { Density } \\
\left(\mathrm{kg} / \mathrm{m}^{3}\right)\end{array}$ & $\begin{array}{l}\text { Viscosity }(\mathrm{cP}) \\
\text { Reference } \\
\text { Measurement }\end{array}$ & $\begin{array}{l}\text { Viscosity } \\
(\mathrm{cP}) \text { MEMS } \\
\text { viscometer }\end{array}$ & $\begin{array}{l}\% \\
\text { Difference }\end{array}$ \\
\hline FBS & 23 & 0.906 & 1.105 & 1.075 & 2.7 \\
FBS & 37 & 0.906 & 0.861 & 0.828 & 3.8 \\
2 mM BSA & 23 & 1.011 & 2.533 & 2.341 & 7.6 \\
2 mM BSA & 37 & 1.011 & 1.808 & 1.815 & 0.4 \\
\hline
\end{tabular}

Table 4

Viscosities of Human Blood Plasma Samples taken from healthy donors. The measurements are conducted with proposed MEMS viscometer at body temperature $\left(37^{\circ} \mathrm{C}\right) . ' \sigma$ ' is the Standard Deviation of 200 data points taken during measurement.

\begin{tabular}{lll}
\hline Donor \# & Viscosity $(\mathrm{cP})$ & $\sigma(\mathrm{cP})$ \\
\hline 1 & 1.280 & 0.017 \\
2 & 1.257 & 0.026 \\
3 & 1.183 & 0.014
\end{tabular}

with $0 \mathrm{M}$ (only FBS), $0.66 \mathrm{mM}, 1.33 \mathrm{mM}, 2 \mathrm{mM}$ BSA were obtained from the MEMS viscometer both at $23^{\circ} \mathrm{C}$ (room temperature) and $37^{\circ} \mathrm{C}$ (body temperature) (Fig. 10). The viscosity increase by the addition of BSA to FBS can be clearly seen.

Also the viscosities $0 \mathrm{M}$ (only FBS) and $2 \mathrm{mM}$ BSA obtained from the MEMS viscometer and reference commercial device are presented in Table 3 . The values both at $23{ }^{\circ} \mathrm{C}$ and $37{ }^{\circ} \mathrm{C}$ are shown. The measurements show a good coherence with a small discrepancy, which can again be explained with the laser heating effect existing in MEMS viscometer.

\subsection{Measurements with human blood plasma}

After the reliability of the system was checked using glycerol and serum measurements, measurements are conducted on plasma taken from healthy donors. After draining blood from each donor in a citrated vacutainer tube, the samples were spun at $5000 \mathrm{rpm}$ for $10 \mathrm{~min}$ in order to separate plasma. The blood plasma viscosities of three different male donors obtained with MEMS viscometer are presented in Table 4. None of the donors has any known diseases showing hyperviscosity symptoms. Their plasma viscosities vary within the range of $1.1 \mathrm{cP}$ and $1.3 \mathrm{cP}$, which is the normal blood plasma viscosity range [2]. Standard deviation of the measurement is also shown in Table 4. This value is noticeably higher than the deviations in glycerol measurements and FBSBSA solutions. This situation is related with the relatively crude and bubbly nature of human blood plasma. It causes local variations of fluid property around the microcantilever and additional noise in optical read-out. Nevertheless, according to these results the system is capable of measuring plasma viscosity with a precision better than $0.03 \mathrm{cP}$. It can be claimed that the device is suitable for screening of patients with hyperviscosity symptoms, having plasma viscosities above $1.8 \mathrm{cP}$.

Blood plasma samples can leave hazardous residues inside the capillary tube of the reference device although it is rinsed after use. Hence the measurements with human blood plasma could not be repeated with the reference viscometer due to this contamination risk. This is a further proof that single use and disposable devices are important for measuring viscosity of biological fluids.

\section{Conclusion}

A new method to measure the blood plasma viscosity with high precision is introduced using a microcantilever. This MEMS-based 
viscometer enables measurement of blood plasma viscosity with a low sample volume requirement $(150 \mu \mathrm{l})$ and can be used in settings where disposable measurement units are a necessity. In the limit this volume can be further reduced to $10 \mu \mathrm{l}$ with appropriate chip design.

Measurements with glycerol solutions with known viscosities are conducted and the response of the system's resonant frequency to viscosity change is obtained. The proposed system is capable of measuring $0.04 \mathrm{cP}$ viscosity difference between $0.86 \mathrm{cP}$ and $3.02 \mathrm{cP}$ which covers the viscosity range of blood plasma. The effect of temperature on viscosity is investigated not only with the MEMS viscometer but also with a reference commercial viscometer. It is seen that the proposed method gives very similar results with the reference measurements with a difference less than $6 \%$ in repeated trials at different times. Measurements with biological liquids are also conducted. The FBS and BSA solutions having viscosities varying between $0.86 \mathrm{cP}$ and $2.53 \mathrm{cP}$ are used. The viscosities of human blood plasma samples taken from healthy donors are measured. The standard deviation in these measurements is lower than $0.03 \mathrm{cP}$. It can be claimed that the MEMS-based viscometer is capable of measuring blood plasma viscosity with high precision and detecting diseases with hyperviscosity symptoms. The system can also be utilized for blood plasma coagulation time measurements, which can be defined as a rapid change of plasma viscosity.

In addition multiplexed measurements can be easily achieved due to the remote nature of actuation and sensing. With the optical readout method of the system, it is possible to perform the readout of multiple sensors simultaneously using an array of optical detectors. Using the proposed method and by combining viscosity and clot time measurements with various agents, a hand-held commercial device with disposable cartridges can be developed.

\section{Acknowledgements}

The authors acknowledge Dr. Necmettin Kilinc for his support during the preparation of biological samples and helpful suggestions, Yasin Kilinc for his help with microfabrication, and Dr. Funda Yagci Acar for her help with the reference measurements. This research is supported by TÜBITAK Grant no. 111E184.

\section{References}

[1] D.H. Lee, J.M. Jung, S.Y. Kim, K.T. Kim, Y.I. Cho, Int. Communi. Heat Mass Transfer 39 (10) (2012) 1474-1477.
[2] G. Késmárky, P. Kenyeres, M. Rábai, K. Tóth, Clin. Hemorheol. Microcir. 39 (1) (2008) 243-246.

[3] H.C. Weng, Y.K. Kao, Apparatus and Method for Measuring Viscosity, Google Patents In., 2011.

[4] N. Antonova, I. Velcheva, Clin. Hemorheol. Microcir. 21 (3/4) (1999) 405-408

[5] B. Holdt, J.K. Lehmann, P. Schuff-Werner, Clin. Hemorheol. Microcir. 33 (4) (2005) 379-388.

[6] J.E. Sader, J. Appl. Phys. 84 (1) (1998) 64-76.

[7] S. Weigert, M. Dreier, M. Hegner, Appl. Phys. Lett. 69 (19) (1996) 2834-2836.

[8] A.I. Romoscanu, M. Sayir, K. Häusler, C. Servais, Measure. Sci. Technol. 14 (4) (2003) 451.

[9] V. Raimbault, D. Rebière, C. Dejous, Mat. Sci. Eng.: C 28 (5) (2008) 759-764.

[10] M. Heinisch, E.K. Reichel, I. Dufour, B. Jakoby, Sens. Actuat. A-Phys. 172 (1) (2011) 82-87.

[11] P.D. Smith, R.C.D. Young, C.R. Chatwin, Measurement 43 (1) (2010) 144-151.

[12] E.K. Reichel, B. Jakoby, C. Riesch, Ieee Sensors vols. 1-3 (2007) (2007) 908-911.

[13] A. Agoston, F. Keplinger, B. Jakoby, Sens. Actuat. A-Phys. 123-24 (2005) 82-86.

[14] M.D. Brouwer, L.A. Gupta, F. Sadeghi, D. Peroulis, D. Adams, Sens. Actuat. APhys. 173 (1) (2012) 102-107.

[15] T.L. Wilson, G.A. Campbell, R. Mutharasan, Sens. Actuat. A-Phys. 138 (1) (2007) 44-51.

[16] U. Sampath, S.M. Heinrich, F. Josse, F. Lochon, I. Dufour, D. Rebiere, IEEE Trans. Ultrason Ferroelectr. Freq. Control 53 (11) (2006) 2166-2173.

[17] C. Vancura, I. Dufour, S.M. Heinrich, F. Josse, A. Hierlemann, Sens. Actuat. APhys. 141 (1) (2008) 43-51.

[18] N. Belmiloud, I. Dufour, A. Colin, L. Nicu, Appl. Phys. Lett. 92 (4) (2008).

[19] M. Youssry, N. Belmiloud, B. Caillard, C. Ayela, C. Pellet, I. Dufour, Sens. Actuat. A-Phys. 172 (1) (2011) 40-46.

[20] I.A.M. Dufour, Y. Amarouchene, C. Ayela, B. Caillard, A. Darwiche, M. Guirardel, H. Kellay EL, F.Mathieu, C. Pellet, D. Saya, M. Youssry, L. Nicu, A. Colin, Journal of Sensors 2012 (2012) 1-9.

[21] P. Rust, J. Dual, Novel method for gated inductive readout for highly sensitive and low cost viscosity and density sensors. in: 16th International Solid-State Sensors, Actuators and Microsystems Conference (Transducers) Beijing, 2011.

[22] P. Rust, I. Leibacher, J. Dual, Temperature Controlled Viscosity and Density Measurements on a Microchip with High Resolution and Low Cost. in: Eurosensors XXV, vol. 25, Amsterdam, 2011.

[23] Y. Zhao, S. Li, A. Davidson, B. Yang, Q. Wang, Q. Lin, J. Micromech. Microeng. 17 (12) (2007) 2528.

[24] X. Huang, S. Li, J.S. Schultz, Q. Wang, Q. Lin, Sens. Actuat. B: Chem. 140 (2) (2009) 603-609.

[25] X. Huang, S. Li, J. Schultz, Q. Wang, Q. Lin, Microelectromech. Syst., J. 18 (6) (2009) 1246-1254.

[26] C. Boss, E. Meurville, J.M. Sallese, P. Ryser, Biosens. Bioelectron. 30 (1) (2011) 223-228.

[27] MicroVisc [http://www.rheosense.com/microvisc.html].

[28] Gluconline [http://electronics.zibb.com/trademark/gluconline/29932920].

[29] E. Timurdogan, N. Ozber, S. Nargul, S. Yavuz, M.S. Kilic, I.H. Kavakli, H. Urey, B.E. Alaca, Biosens. Bioelectron. 26 (1) (2010) 195-201.

[30] E. Timurdogan, B.E. Alaca, I.H. Kavakli, H. Urey, Biosens. Bioelectron. 28 (1) (2011) 189-194.

[31] J.W.M. Chon, P. Mulvaney, J.E. Sader, J. Appl. Phys. 87 (8) (2000) 3978-3988.

[32] A. Maali, C. Hurth, R. Boisgard, C. Jai, T. Cohen-Bouhacina, J.P. Aime, Hydrodynamics of oscillating atomic force microscopy cantilevers in viscous fluids, J. Appl. Phys. 97 (7) (2005).

[33] O. Cakmak, C. Elbuken, E. Ermek, S. Bulut, Y. Kilinc, I. Baris, H. Kavakli, E. Alaca, H. Urey, New Biotechnol. 29 (2012) S162-S163. 